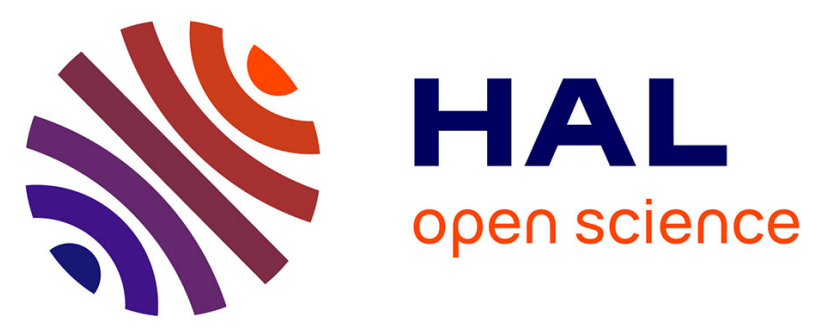

\title{
Physical properties and giant magnetoimpedance sensitivity of rapidly solidified magnetic microwires
}

Basile Dufay, Sébastien Saez, Christophe Dolabdjian, Arthur Yelon, David Ménard

\section{- To cite this version:}

Basile Dufay, Sébastien Saez, Christophe Dolabdjian, Arthur Yelon, David Ménard. Physical properties and giant magnetoimpedance sensitivity of rapidly solidified magnetic microwires. Journal of Magnetism and Magnetic Materials, 2012, 324 (13), pp.2091-2099. 10.1016/j.jmmm.2012.02.012 . hal-00746982

\section{HAL Id: hal-00746982 \\ https://hal.science/hal-00746982}

Submitted on 30 Oct 2012

HAL is a multi-disciplinary open access archive for the deposit and dissemination of scientific research documents, whether they are published or not. The documents may come from teaching and research institutions in France or abroad, or from public or private research centers.
L'archive ouverte pluridisciplinaire $\mathbf{H A L}$, est destinée au dépôt et à la diffusion de documents scientifiques de niveau recherche, publiés ou non, émanant des établissements d'enseignement et de recherche français ou étrangers, des laboratoires publics ou privés. 


\title{
Physical properties and giant magnetoimpedance sensitivity of rapidly solidified magnetic microwires
}

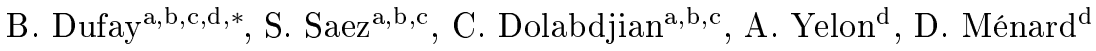 \\ ${ }^{a}$ Université de Caen Basse-Normandie, UMR 6072 GREYC, F-14032 Caen, France \\ ${ }^{b}$ ENSICAEN, UMR 6072 GREYC, F-14050 Caen, France \\ ${ }^{c}$ CNRS, UMR 6072 GREYC, F-14032 Caen, France \\ ${ }^{d}$ École Polytechnique de Montréal, département de génie physique $\mathscr{E}$ regroupement québécois des matériaux de pointe, \\ Montréal, Québec, Canada H3C3A7
}

\begin{abstract}
The relation between the magnetoimpedance and the magnetic properties of a wide set of soft magnetic microwires from several sources has been studied. Magnetic properties were obtained by vibrating sample magnetometry and ferromagnetic resonance spectroscopy. The magnetoimpedance voltage sensitivity of each sample, the criterion of interest for high sensitivity magnetometer design, was then evaluated at several frequencies and drive currents. It appears that all samples possess roughly similar properties, regardless of their fabrication process or chemical composition. The voltage sensitivity of the samples obtained from experimental measurement is compared with a simple model of sensitivity. The general trends predicted by the model provide useful insights for materials optimization. Averaged sensitivity over the sample set is around $10 \mathrm{kV} / \mathrm{T} / \mathrm{cm}$ at $10 \mathrm{MHz}$. The critical importance for sensitive magnetometry of the maximum excitation current permissible in each wire is also highlighted.
\end{abstract}

Keywords: Giant Magnetoimpedance (GMI), Voltage Sensitivity, Magnetometry, Soft Amorphous Wires, Ferromagnetic resonance, Magnetic Sensors.

\section{Introduction}

The giant magnetoimpedance (GMI) effect has attracted great attention in the past two decades, due to its potential for the design of low cost, high performance, magnetometers [1]. Much of the effort toward optimization of GMI materials has been directed towards increasing the amplitude of the relative impedance variation with respect to the external applied magnetic field,

$$
\frac{\Delta Z}{Z_{r e f}}=\frac{Z-Z_{r e f}}{Z_{r e f}}
$$

in relation to a reference value, $Z_{\text {ref }}$. The latter is generally chosen to be the impedance at zero field, or at the maximum available field. As was recently discussed [2], this GMI ratio, Eq. (1), is not particularly meaningful as a metric for sensitive magnetometry and it can be misleading in the comparison

\footnotetext{
*Corresponding author

Email address: basile.dufay@unicaen.fr (B. Dufay)
}

of the performance between GMI wires from different sources.

Here, we adopt the pragmatic point of view that the main criterion relevant to the design of highly sensitive GMI magnetometers is the maximum voltage sensitivity, defined as the differential variation of voltage across the GMI sample, divided by the applied magnetic field, expressed in $\mathrm{V} / \mathrm{T}[2,3]$. This value is related to the differential variation in impedance, which we may also call the intrinsic impedance sensitivity, $S_{\Omega / \mathrm{T}_{\max }}=\partial Z /\left.\partial B\right|_{B=B_{o p t}}$, evaluated under optimal bias field, $B_{\text {opt }}\left(=\mu_{0} H_{\text {opt }}\right)$, and optimal AC drive current, $I_{A C-o p t}$, permissible in the wire. We emphasize that, in order to be meaningful, the response must be measured under optimal bias and driving conditions. In high sensitivity magnetometer design, a high signal-tonoise ratio (SNR) is extremely important. Thus, a voltage sensitivity as high as possible is clearly desirable whenever the dominant noise source is that of the electronic conditioning circuitry, as has been 


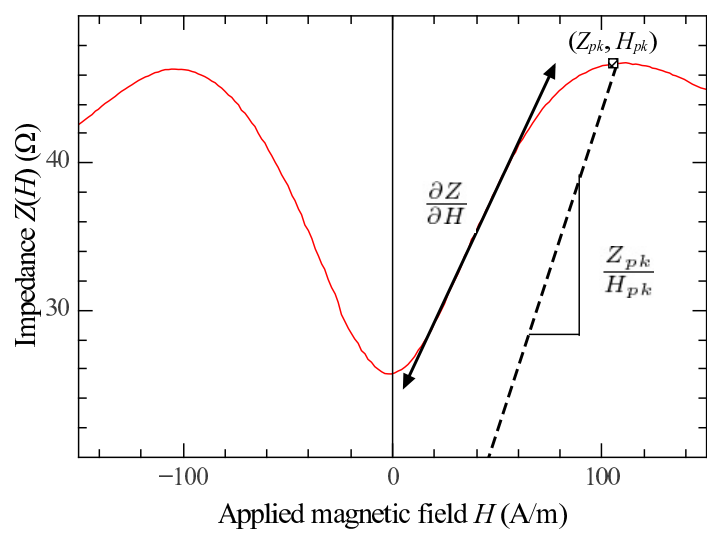

Figure 1: Modulus of the impedance variation versus applied magnetic field, from [2]. The black solid straight line represents the maximum slope, that is the intrinsic impedance sensitivity. The dotted line is the approximation for slope used in the simple model of sensitivity.

observed $[3,4]$.

Ménard and collaborators [2] have proposed a model in order to establish simple relations between GMI sensitivity and material parameters, along with driving current and frequency. The maximum voltage sensitivity, in units of $\mathrm{V} / \mathrm{T}$, is reached at the optimal driving current and external bias field. It is estimated from

$$
\begin{aligned}
S_{\mathrm{V} / \mathrm{T}_{\max }} & =S_{\mathrm{V} / \mathrm{T}}\left(I_{A C-o p t}, B_{o p t}\right) \\
& =I_{A C-o p t} S_{\Omega / \mathrm{T}_{\max }} \approx I_{A C-o p t} \frac{Z_{p k}}{\mu_{0} H_{p k}} .
\end{aligned}
$$

That is, it was approximated as the ratio of maximum impedance value, $Z_{p k}$, over its field position, $H_{p k}$, as illustrated in Fig. 1. In the strong skin effect regime, for a monodomain soft magnetic wire, of length $l$, and with a perfectly circumferential anisotropy within the skin depth region, the voltage sensitivity, per unit length, may be estimated using [2]

$$
\begin{aligned}
& \frac{S_{\mathrm{V} / \mathrm{T}_{\max }}}{l} \approx\left(\frac{M_{s}^{2} \omega \rho^{3}}{\mu_{0}^{2} A}\right)^{1 / 4}\left[\frac{H_{\text {crit }}(\omega)}{H_{p k}(\omega)}\right]\left(\omega \leq \omega_{c}\right) \\
& \frac{S_{\mathrm{V} / T_{\max }}}{l} \approx\left(\frac{\gamma M_{s} \rho}{\alpha}\right)^{1 / 2}\left[\frac{H_{\text {crit }}(\omega)}{H_{p k}(\omega)}\right]\left(\omega \geq \omega_{c}\right)
\end{aligned}
$$

where $\omega$ is the excitation angular frequency, $M_{s}$ is the saturation magnetization of the sample, $\rho$ is its resistivity, $\alpha$ is the Gilbert damping parameter, $\gamma$ is the gyromagnetic ratio, $A$ is the exchange stiffness constant (approximately $10^{-11} \mathrm{~J} / \mathrm{m}$ ) and
$H_{c r i t}$ is the critical surface magnetic field related to the optimal driving current, $I_{A C-o p t}=2 \pi a H_{c r i t}$. The angular frequency, $\omega_{c}$, is the crossover frequency, above which the maximum impedance is no longer limited by exchange-conductivity effects (equation (3a)), but by phenomenological Gilbert damping (equation (3b)). The field ratio, $H_{c r i t} / H_{p k}$, is on the order of unity for ideal wires in the quasi-static regime. At higher frequency, both fields increase with increasing frequency, but $H_{c r i t}$ increases faster than $H_{p k}$. In what follows, we assume conservatively that this field ratio is roughly unity in the frequency range considered.

While Eqs. (3a) and (3b) are likely to be too simple to comprehend all the subtleties of the GMI effect, they provide useful design rules for material and sensor optimization. Indeed, the voltage sensitivity per unit length, measured under optimal driving condition, is a material-dependent and radiusindependant metric to gauge the merit of different samples. It is also important to note that for real (non-ideal) wires, which do not exhibit perfect circumferential anisotropy, the maximum impedance variation is more likely to be described by an effective Gilbert damping parameter, corresponding to a sensitivity given by (3b) at all frequencies.

Here, we confront this simple model by analyzing and comparing a set of different GMI microwires, fabricated in different laboratories. The emphasis is placed on the relation between voltage sensitivities and physical properties. This required us to measure the physical properties of the wires. Therefore, a significant part of the discussion is dedicated to the proper determination of the relevant physical properties (material parameters) of rapidly solidified microwires. Section 2 describes the experimental details and data analysis. The measured physical properties are presented in Sec. 3. Sensitivity performances are discussed and compared in Sec. 4, which is followed by a general conclusion.

\section{Experimental}

\subsection{Materials and sample preparation}

A set of ten different samples of soft amorphous microwires with different chemical compositions (essentially based on CoFeSiB alloys except for sample a2) and made by three different fabrication processes were selected for this study. The samples are described in Table 1. The first three samples, a1 to a3, were obtained by melt-extraction [5] and 
were provided by MXT, in Montréal. Samples b1 to b4 are glass-covered amorphous wires [6] with a thin glass coating, provided by the department of Fisica de Materiales UPV/EHU (San Sebastian). Samples c1 to c3, from the Institute of R\&D for Technical Physics (Iasi, Romania), consist of two glass-covered wires with thick glass coating and one with large radius, obtained by quenching in rotating water [7]. The exact composition of the alloys from the third provider is unknown. Let us emphasize that the samples were chosen to provide a variety of fabrication methods and physical properties, but were not necessarily optimized for GMI applications. A permalloy and a metglas microwire, from MXT, part of the original set, are not included due to their poor GMI behavior.

In order to compare the measured GMI sensitivity to the results of the model outlined in the introduction, we have measured the following relevant physical properties: the saturation magnetization, $M_{s}$, the resistivity, $\rho$, the Gilbert damping parameter, $\alpha$, and the Landé-factor, $g=\gamma \hbar / \mu_{B}$ where $\gamma$ is the gyromagnetic ratio, $\mu_{B}$ the Bohr magneton and $\hbar$ the reduced Planck constant. In addition, we measured the coercive magnetic field, $H_{C}$, as an indicator of the soft magnetic behavior. The samples were also characterized regarding their intrinsic impedance sensitivity, $S_{\Omega / \mathrm{T}}\left(\mu_{0} H\right)$, and voltage sensitivity, $S_{\mathrm{V} / \mathrm{T}}\left(I_{A C}, \mu_{0} H\right)$.

First, a $7 \mathrm{~cm}$ segment of each material was cut for determination of its DC resistance per unit length, using a 4-point measurement setup. Radius, $a$, was estimated using optical microscopy. For samples b1 to c3, a nominal radius was also provided by the supplier. Values of the resistances, lengths and diameters yielded the electrical resistivity.

Each $7 \mathrm{~cm}$ segment was subsequently cut into three parts. The first, about $0.5 \mathrm{~cm}$ long, was used for vibrating sample magnetometry (VSM), the second, less than $0.2 \mathrm{~cm}$ long, was dedicated to ferromagnetic resonance measurement (FMR). The remaining segment was cut into $1.5 \mathrm{~cm}$ long samples, which were soldered onto a sample holder for determination of GMI response. As the estimation of radii by optical microscopy was found to be imprecise, we used the magnetic characterization to derive the diameter, as explained below. The following subsections explain the various characterization procedures.

\subsection{Vibrating Sample Magnetometry (VSM)}

The static magnetic properties were investigated using a vibrating sample magnetometer (VSM). We obtained the magnetic moment as a function of external applied field for each sample for various angles, $\theta_{H}$, between the applied field and the wire axis. In Fig. 2, we show a representative curve of the normalized magnetization as a function of field for parallel, $\theta_{H}=0$, and perpendicular, $\theta_{H}=\frac{\pi}{2}$, fields (sample a1). For each sample, we measured the longitudinal coercive field, $H_{C}$, and the normalized apparent susceptibility (slope $\left.\partial\left(M / M_{s}\right) /\left.\partial H\right|_{M=0}\right)$, $\chi_{\|}$and $\chi_{\perp}$, for both magnetization directions as illustrated in Fig 2. Only the parallel and perpendicular field results were retained for this work.

The VSM was calibrated using a Ni disk as reference. The saturation magnetization, $M_{s}$, may be estimated from the maximum value of the measured magnetic moment, $|\vec{m}|$, from

$$
M_{s}=\frac{|\vec{m}|}{l \pi a^{2}}
$$

where $l \pi a^{2}$ represents the volume of the sample under measurement. While this is the most direct evaluation of the saturation magnetization, uncertainties as to the length and, mainly, the diameter place limits on its usefulness.

Another estimate can be obtained by extrapolating the linear portion of the magnetization curve to saturation, and assuming that the field at saturation is equal to the demagnetization field, $M_{s} / 2$. An alternative version of this approach was recently proposed [8] for samples for which the magnetization processes are dominated by the dipolar fields. It is assumed that such samples should exhibit apparent initial susceptibilities, $\chi_{i}$, given by

$$
M_{i}=\chi_{i} H=\chi_{0}\left(H-N_{i} M_{i}\right),
$$

where $\chi_{0}$ is an isotropic intrinsic susceptibility, $i=\|$ or $\perp$ and $N_{i}$ is the demagnetization factor. Assuming that $N_{\|} \chi_{\|} \ll 1$, which implies that $N_{\perp}$ is approximately $1 / 2$, then

$$
\begin{aligned}
\chi_{\|} & \approx \chi_{0}, \\
\chi_{\perp} & \approx \chi_{0}\left(1-\frac{1}{2} \chi_{\perp}\right) .
\end{aligned}
$$

Defining a normalized susceptibility, $\overline{\chi_{i}}=\chi_{i} / M_{s}$, and combining Eqs. (6a) and (6b),

$$
\overline{\chi_{\perp}}=\overline{\chi_{\|}}\left(1-\frac{1}{2} \overline{\chi_{\perp}} M_{s}\right) .
$$


Table 1: Sample description.

\begin{tabular}{ccccc}
\hline Sample & Composition & Fabrication & Diameter $2 a$ & Other \\
\hline \hline a1 & $\mathrm{Co}_{70.54} \mathrm{Fe}_{3.95} \mathrm{Si}_{15.91} \mathrm{~B}_{7.13} \mathrm{Nb}_{2.91}$ & melt extracted & $35 \mu \mathrm{m}^{\mathrm{ii}}$ & \\
$\mathrm{a} 2$ & $\mathrm{Ni}_{45} \mathrm{Co}_{25} \mathrm{Fe}_{6} \mathrm{Si}_{9} \mathrm{~B}_{13} \mathrm{Mn}_{2}$ & melt extracted & $17 \mu \mathrm{m}^{\mathrm{ii}}$ & $\mathrm{Ni}$ rich \\
$\mathrm{a} 3$ & $\mathrm{Co}_{71} \mathrm{Fe}_{4} \mathrm{Si}_{13.5} \mathrm{~B}_{6.5} \mathrm{Nb}_{5}$ & melt extracted & $35 \mu \mathrm{m}^{\mathrm{ii}}$ & \\
b1 & $\mathrm{Co}_{67} \mathrm{Fe}_{3.9} \mathrm{~B}_{11.5} \mathrm{Si}_{14.5} \mathrm{Ni}_{1.5} \mathrm{Mo}_{1.6}$ & thin glass-covered & $21.4 \mu \mathrm{m}^{\mathrm{iii}}$ & $2.4 \mu \mathrm{m}$ glass \\
b2 & $\mathrm{Co}_{67} \mathrm{Fe}_{3.9} \mathrm{~B}_{11.5} \mathrm{Si}_{14.5} \mathrm{Ni}_{1.5} \mathrm{Mo}_{1.6}$ & thin glass-covered & $16.4 \mu \mathrm{m}^{\mathrm{iii}}$ & $1.5 \mu \mathrm{m}$ glass \\
b3 & $\mathrm{Co}_{67} \mathrm{Fe}_{3.9} \mathrm{~B}_{11.5} \mathrm{Si}_{14.5} \mathrm{Ni}_{1.5} \mathrm{Mo}_{1.6}$ & thin glass-covered & $25.6 \mu \mathrm{m}^{\mathrm{iii}}$ & $0.5 \mu \mathrm{m}$ glass \\
b4 & $\mathrm{Co}_{66} \mathrm{Fe}_{4} \mathrm{~B}_{14} \mathrm{Si}_{15} \mathrm{Ni}_{1}$ & thin glass-covered & $22.6 \mu \mathrm{m}^{\mathrm{iii}}$ & $2.0 \mu \mathrm{m}$ glass \\
c1 & $\mathrm{CoFeSiB}_{\mathrm{iii}}$ & thick glass-covered & $20 \mu \mathrm{m}^{\mathrm{ii}}$ & $13.5 \mu \mathrm{m}$ glass \\
c2 & $\mathrm{CoFeSiB}$ & thick glass-covered & $27 \mu \mathrm{m}^{\mathrm{iii}}$ & $21.5 \mu \mathrm{m}$ glass \\
c3 & $\mathrm{CoFeSiB}$ & rotating water & $100 \mu \mathrm{m}^{\mathrm{iii}}$ & Large radius \\
\hline
\end{tabular}

\footnotetext{
${ }^{\mathrm{i}}$ Exact compositions of samples $\mathrm{c} 1$ to $\mathrm{c} 3$ are unknown.

ii Estimated value from optical microscopy.

iii Nominal value provided by the supplier.
}
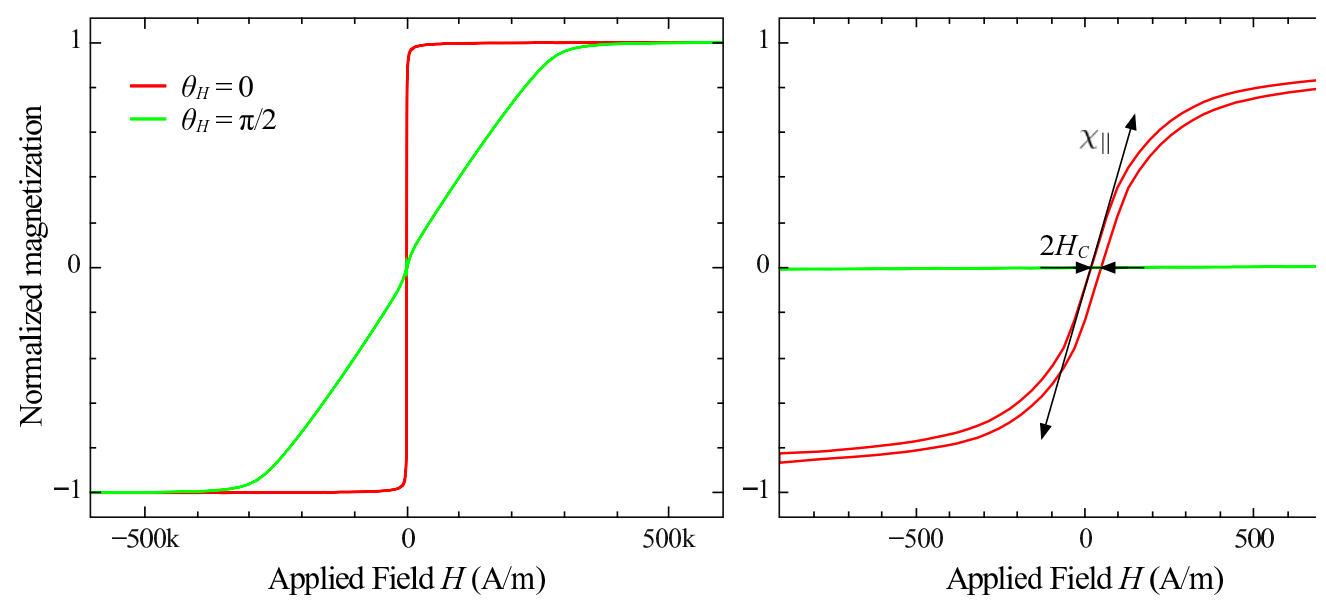

Figure 2: Normalized magnetization loops of the sample a1 for two applied field angle, $\theta_{H}=0$ and $\theta_{H}=\frac{\pi}{2}$. It illustrates deduced characteristics, $H_{C}$, and $\chi_{\|}$. Note that the field scale of the two images differs by approximately 1000 . 
Solving Eq. (7) for $M_{s}$, we find

$$
M_{s}=2 \frac{1-\overline{\chi_{\perp}} / \overline{\chi_{\|}}}{\overline{\chi_{\perp}}},
$$

which provides an experimental value for the magnetization, independent of our knowledge of the sample volume. For ultra-soft wires with small diameter-to-length ratio, the above analysis simplifies to assuming an apparent perpendicular susceptibility of $2\left(1 / N_{\perp}\right.$ with $\left.N_{\perp}=1 / 2\right)$, so that the saturation magnetization is approximately $2 \overline{\chi_{\perp}}$. As a corollary, combining Eqs. (8) and (4) yields an estimation of the magnetic volume. Since the length and the magnetization of the samples are measured with a relatively fair accuracy, the procedure thus leads to experimental values of the wire radii, which are far more reliable than the values obtained from optical microscopy.

VSM measurements were also used to investigate the approach to saturation for each sample. This can be related to imperfections of the materials and may serve as a discriminant between samples. For ideal samples with uniaxial anisotropy, approaching saturation by coherent rotation, we would expect a law of the form $M(H)=M_{s}\left(1-b / H^{2}\right)$ [9], where $b$ is related to the anisotropy constant and direction of the applied field relative to the anisotropy axis. However, in real (non-ideal) materials, it is common to observe behavior following forms such as $M(H)=M_{s}\left(1-c / H^{\beta}-\ldots\right)$ where the exponent $\beta$ is related to various defects of the materials [1012]. The parameter $\beta$ thus provides an indication of "non-ideality" of the samples. By rewriting the $M(H)$ formula as $\log \left(1-M / M_{s}\right)=\log c-\beta \log H$, one may obtain the power law from the slope of the logarithmic plot of $\left(1-M / M_{s}\right)$ versus $1 / H$ as illustrated in Fig. 3. All of the observed values (Table 2) range between $1 / 2$ and 1 .

\subsection{Ferromagnetic Resonance measurement (FMR)}

The microwave response of each sample was investigated in order to observe the ferromagnetic resonance (FMR) and ferromagnetic antiresonance (FMAR). Spectra were obtained at room temperature for different field angles using the same equipment and procedure described in [8], using a cylindrical resonant cavity at $38 \mathrm{GHz}$. We focus here on axially $\left(\theta_{H}=0\right)$ magnetized wires. Microwires were placed in a region with nearly zero dynamic electric field and maximum dynamic magnetic field,

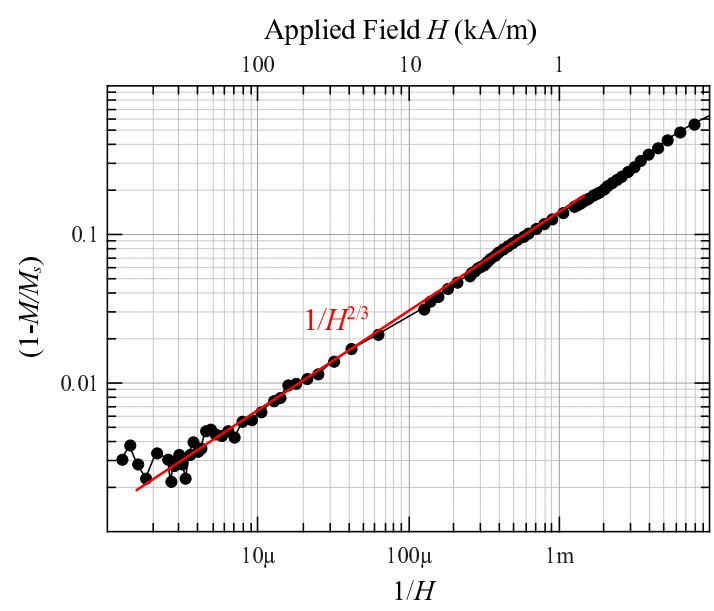

Figure 3: Evaluation of the exponent of the law-of-approachto-saturation from magnetization curve of the sample a1, obtained by VSM measurement.

linearly polarized transverse to the wire axis. From the spectra of axially magnetized wires, we deduced the FMR field, $H_{r}$, FMAR field, $H_{a r}$, and resonant linewidth, $\Delta H$. Figure 4 illustrates the extraction of these parameters for sample a1. For microwires, it has been shown [13] that the skin effect combined with dipolar interactions yields a FMR field position given by the Kittel equation [14, 15] for in-plane static field and insulating thin films, and the FMAR is given, for the same conditions, by Van Vleck [15]

$$
\begin{aligned}
\left(\frac{\omega}{\gamma \mu_{0}}\right)^{2} & =H_{r}\left(H_{r}+M_{s}\right), \\
\frac{\omega}{\gamma \mu_{0}} & =H_{a r}+M_{s} .
\end{aligned}
$$

From the FMR and FMAR fields at a given frequency, one can deduce the saturation magnetization, $M_{s}$, and the gyromagnetic ratio, $\gamma$. In contrast, the FMR linewidth, which is a measure of magnetic losses, strongly depends upon microwire properties. It is linked to the Gilbert damping parameter, $\alpha$, and to eddy current losses. A comprehensive analysis of the FMR response of microwires in this experimental configuration was recently proposed [16]. Here, the Gilbert damping parameters were deduced by fitting the FMR experimental curves based on the analysis of ref. [16].

\subsection{Magnetoimpedance}

Magnetoimpedance, $Z(H)$, in the linear regime was measured using a network analyzer as described 


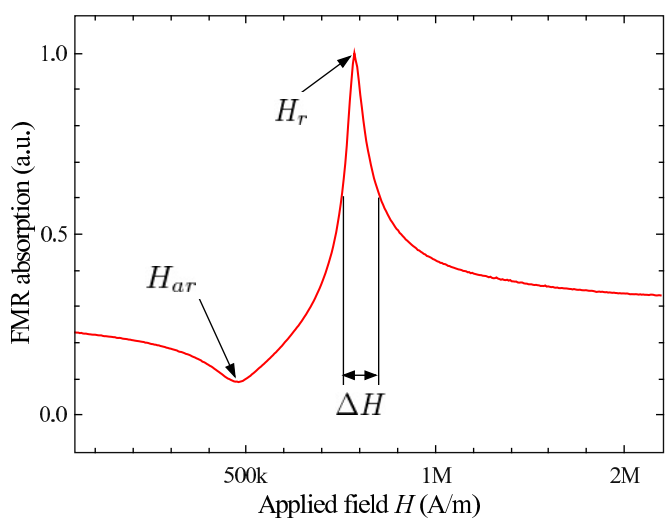

Figure 4: Ferromagnetic resonance absorption spectrum of the sample a1. The extraction of $H_{r}, H_{a r}$, and $\Delta H$ from this measurement are illustrated.

in [17]. Measurements were obtained for several DC bias currents, $I_{D C}$, (from -10 to $10 \mathrm{~mA}$ ) and several excitation frequencies, $f(3 \mathrm{MHz}$ to $300 \mathrm{MHz})$. Intrinsic impedance sensitivity, expressed as Ohms per Tesla, $S_{\Omega / \mathrm{T}}\left(\mu_{0} H\right)$, in the magnetic field range, was then evaluated by numerical differentiation, as shown in Fig. 5. The use of a DC bias current results in an effective field with helical symmetry, which produces the observed asymmetric response. Measurements in the linear regime enabled us to determine the optimal DC bias current, which maximizes the intrinsic impedance sensitivity, for each sample. This current was then used in the measurement of the voltage sensitivity as described below.

The simplified model of the sensitivity presented in Eqs. (3a) and (3b) assumes that the sample is operated under an optimal excitation current amplitude, above which the sensitivity begins to decrease. Both experiment and calculation [18] show that the value of this optimal excitation current is slightly larger than the value for the onset of nonlinear behavior, and tends to increase with increasing operating frequency.

Consequently, the voltage sensitivity was evaluated under various sine excitation current amplitudes, $I_{A C}$, by numerical differentiation of the measured voltage, $V(H)$, at the sample terminals, versus applied magnetic field. The setup used is described in [18]. Wires were subjected to the optimal DC bias current deduced from intrinsic impedance sensitivity characterization. Excitation current frequency, $f$, was varied from $300 \mathrm{kHz}$ to $10 \mathrm{MHz}$.

\section{Results: physical properties}

The measured physical properties of each sample are summarized in Table 2. All samples exhibit typical values for soft magnetic metals. The diameters were determined from the magnetic volume, as discussed in Section 2.2. While some discrepancies are observed as compared to nominal or optically measured values, one must keep in mind that fluctuations of diameters are common in soft amorphous microwires. The presence of such fluctuations is also suggested by our electrical resistance measurements of different pieces of microwires of the same composition, which exhibits significant variations. The resistances reported in Table 2 refer to $7 \mathrm{~cm}$ long samples and are not necessarily always representative of the resistance of the smaller wires cut from it and used in the GMI experiments. With this in mind, values reported for the resistivity, calculated from the estimated resistance and diameters, are, therefore, not always very precise. If we consider the fluctuations of the DC resistance and the geometrical parameters, the level of confidence in the resistivity evaluation is not better than $20 \%$.

Saturation magnetization was obtained both from VSM data (Eq. (8)) and FMR data (Eqs. (9a) and $(9 b))$. As anti-resonance was not observed in the FMR spectra for sample b1 to b4, the Landé factor could not be determined. The saturation magnetization for these samples was thus determined using Eq. (9a) with an assumed Landé factor of 2.12 , corresponding to the average value of the other samples with similar composition. Gilbert parameters, $\alpha$, are all between 0.01 and 0.02 , which is also on the order expected for such materials.

Evaluation of the saturation magnetization from Eqs. (8) (VSM) and (9a)-(9b) (FMR) are in a fairly good agreement. This is illustrated in Fig. 6 which compares the different values obtained for each sample. In contrast, $M_{s}$ determined using Eq. (4), from the saturated magnetic moment measured in the VSM and the diameters reported in Table 1, tends to be considerably lower. This is another indication that the nominal or optically measured diameters might be misleading. In what follows, we use the value of $M_{s}$ obtained from Eq. (8) (VSM), close to that of Eqs. (9a) and (9b) (FMR).

As expected for soft magnetic materials, the susceptibilities are high and the coercive fields are low (smaller than $4 \mathrm{~A} / \mathrm{m}$ ) except for samples a1 and a2 for which $H_{C}$ is greater than $15 \mathrm{~A} / \mathrm{m}$. We note that both the susceptibility and coercive fields are struc- 

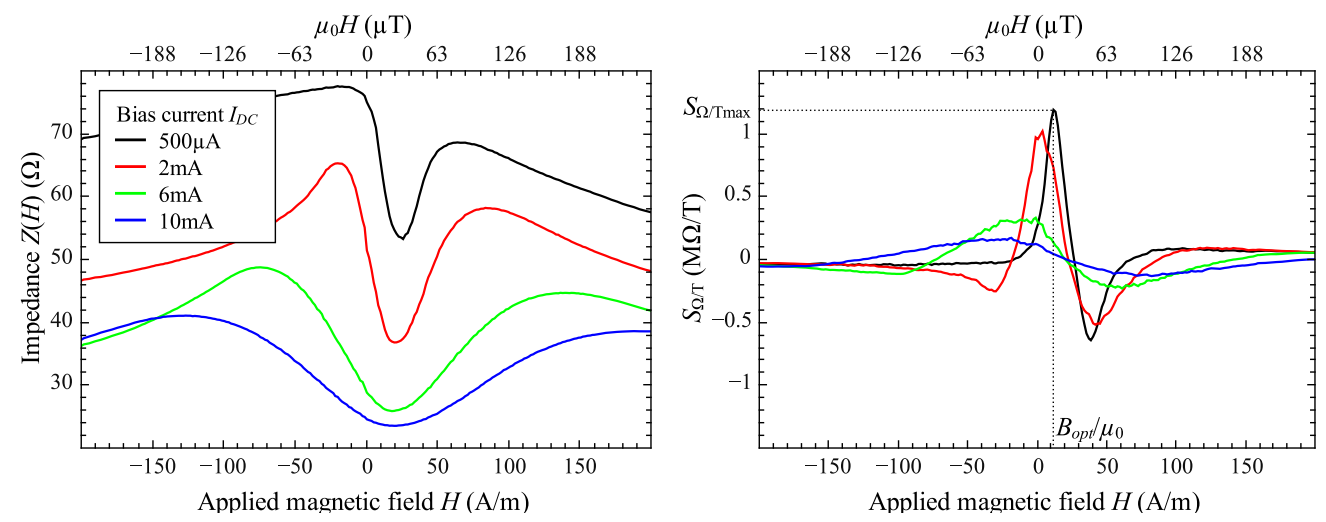

Figure 5: Impedance variation, $Z(H)$, versus applied magnetic field for sample a1, measured using a vector network analyzer at an excitation frequency of $3 \mathrm{MHz}$ and several DC bias currents, $I_{D C}$. Maximum intrinsic impedance sensitivity, $S_{\Omega / \mathrm{T}_{\text {max }}}$, is determined by numerical differentiation.

Table 2: Measured physical properties of each sample.

\begin{tabular}{|c|c|c|c|c|c|c|c|c|c|c|}
\hline Sample & $\begin{array}{l}2 a^{\mathrm{i}} \\
\mu \mathrm{m}\end{array}$ & $\begin{array}{l}R_{D C} / l \\
\Omega / \mathrm{cm}\end{array}$ & $\begin{array}{c}M_{s}(\mathrm{VSM})^{\mathrm{ii}} \\
\mathrm{kA} / \mathrm{m}\end{array}$ & $\begin{array}{c}M_{s}(\mathrm{FMR})^{\mathrm{iii}} \\
\mathrm{kA} / \mathrm{m}\end{array}$ & $\chi_{\|}$ & $\alpha$ & $g$ & $\begin{array}{c}\rho \\
\mu \Omega . c m\end{array}$ & $\begin{array}{c}H_{C} \\
\mathrm{~A} / \mathrm{m}\end{array}$ & $\beta$ \\
\hline a1 & 35.8 & 12.7 & 558 & 549 & 2520 & 0.011 & 2.10 & 128 & 15.9 & $2 / 3$ \\
\hline $\mathrm{a} 2$ & 15.3 & 40 & 446 & 445 & 11640 & 0.010 & 2.12 & 74 & 15.9 & $2 / 3$ \\
\hline a3 & 30.0 & 19.4 & 548 & 561 & 2700 & 0.012 & 2.12 & 137 & 3.6 & $2 / 3$ \\
\hline b1 & 19.3 & 40 & 507 & $475^{\text {iv }}$ & 2910 & 0.015 & $2.12^{\mathrm{v}}$ & 118 & 3.6 & $1 / 2$ \\
\hline b2 & 12.7 & 65.7 & 473 & $454^{\text {iv }}$ & 2480 & 0.014 & $2.12^{\mathrm{v}}$ & 83 & 2.0 & $1 / 2$ \\
\hline b3 & 24.2 & 37.1 & 442 & $475^{\text {iv }}$ & 4030 & 0.012 & $2.12^{\mathrm{v}}$ & 171 & 3.2 & $1 / 2$ \\
\hline b4 & 20.8 & 38.6 & 489 & $496^{\mathrm{iv}}$ & 1600 & 0.013 & $2.12^{\mathrm{v}}$ & 131 & 2.8 & $1 / 2$ \\
\hline $\mathrm{c} 1$ & 17.8 & 42.9 & 552 & 598 & 4160 & 0.011 & 2.11 & 107 & 3.6 & $2 / 3$ \\
\hline $\mathrm{c} 2$ & 26.4 & 23.7 & 566 & 560 & 2540 & 0.012 & 2.15 & 130 & 2.4 & $1 / 2$ \\
\hline $\mathrm{c} 3$ & 102 & 1.6 & 542 & 561 & 200 & 0.020 & 2.11 & 129 & 2.4 & 1 \\
\hline
\end{tabular}

\footnotetext{
${ }^{\mathrm{i}}$ Magnetic diameter.

ii Estimated from equation (8).

iii Estimated from equations (9a) and (9b).

${ }^{i v}$ Considering an assumed value of $g=2.12$.

${ }^{v}$ Assumed value.
} 


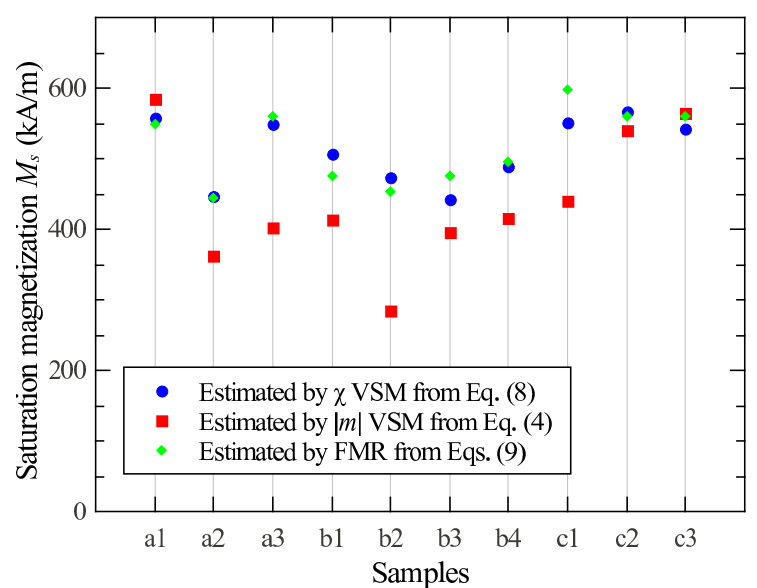

Figure 6: Measured saturation magnetization values for each sample from three methods.

ture sensitive properties, which may be affected not only by the composition and fabrication method, but also by the residual strains or the presence of defects which may be introduced during fabrication or during mounting for measurement. Likewise, the anisotropy is affected by the strains of fabrication and mounting. In the case of glass-covered wires, the glass and metal exert strains on each other.

Finally, three different power law exponents for approach to saturation of the magnetization have been obtained, as shown in the last column of table 2 . Departures from an ideal $1 / H^{2}(\beta=2)$ [9] law for uniaxial amorphous materials may be interpreted as due to inhomogeneities in composition, or induced by magnetoelastic interactions with inhomogeneous stresses [12], or to surface roughness [19]. This exponent appears to be somewhat correlated to source and preparation method.

Except for sample a2, which has a composition significantly different from the others, most CoFebased samples exhibit similar properties, yet there are variations, which cannot be accounted for by measurement uncertainties. For instance, an average resistivity around $130 \mu \Omega . c m$ is generally observed. Exceptions are sample b2 which shows an unusually low value, of $83 \mu \Omega . c m$, and samples $\mathrm{c} 1$ $(107 \mu \Omega . c m)$ and b3 (171 $\mu$.cm) which are somewhat lower and higher than average. The saturation magnetization also exhibits small variations from sample to sample. The b-series (nominally with identical compositions) have average $M_{s}$ of $480 \mathrm{kA} / \mathrm{m}$, significantly smaller than the values of the other CoFe-rich samples which are around
$560 \mathrm{kA} / \mathrm{m}$. The average phenomenological Gilbert damping parameter is approximately 0.012 (except for the larger wire $\alpha=0.020$ ) and Landé factors are all slightly higher than 2, regardless of the fabrication process and chemical composition. As pointed out in the introduction, the sensitivity of non ideal wires in the $\mathrm{MHz}$ regime is likely to be described by an effective Gilbert damping parameter in Eq. (3b), which could be significantly higher than that which is measured in the $\mathrm{GHz}$ regime. Nevertheless, $\alpha$ remains a meaningful indicator of the microstructural quality of the samples.

\section{Measured and theoretical voltage sensi- tivity}

The voltage sensitivity of each sample was measured as described in section 2.4. There might be difficulties in GMI measurements, associated with the reproducibility of electrical contacts and with the variable mechanical stresses introduced during the contact procedure. The maximum RMS value of the excitation current flowing through the wire was limited to $10 \mathrm{~mA}_{\mathrm{rms}}$. Note that for some samples the optimal excitation current is greater than this value so that the optimal driving current was not reached. For such samples the maximum voltage sensitivity is underestimated.

Figure 7 shows the highest voltage sensitivity of each sample as a function of excitation current amplitude for an operating frequency of $3 \mathrm{MHz}$. At this frequency, samples a3, b1 and b3 outperform the others with sensitivities around $7 \mathrm{kV} / \mathrm{T} / \mathrm{cm}$, whereas samples a2 and b4 yield relatively poor sensitivity. The optimal driving currents for samples a2 and c3 are clearly higher than the maximum employed in this study. In all cases, intrinsic sensitivity depends upon the operating conditions. A sample will be better or worse than another, depending upon the driving current chosen. Any comparison between specimens which does not properly account for this is rendered less meaningful.

The maximum measured voltage sensitivity for each sample is presented in Table 3 for several frequencies. Not surprisingly, the voltage sensitivity increases with frequency. At lower frequencies, sample a3 outperforms the others, but is surpassed by sample b1 at $3 \mathrm{MHz}$. Values in italics correspond to those for which the optimal driving current amplitude is not reached (greater than $10 \mathrm{~mA}$ ). As theoretically expected, this value increases with frequency. At $10 \mathrm{MHz}$ half of the samples operate 
below their optimal sensitivity and two samples, a2 and $\mathrm{c} 3$, have their optimal driving currents above $10 \mathrm{~mA}$ at all frequencies considered.

Figure 8 compares three different ways of expressing the sensitivity at $3 \mathrm{MHz}$. The sample list of the abscissa is ordered by increasing maximum intrinsic impedance sensitivity. First, it compares the maximum intrinsic impedance sensitivity, $S_{\Omega / \mathrm{T}_{\max }}$ (curve (a)), obtained in the linear regime, with the voltage sensitivity (curve (b)) measured at low excitation current, $S_{\mathrm{V} / \mathrm{T}}\left(I_{a c}=1 \mathrm{~mA}_{\mathrm{rms}}, B_{o p t}\right)$. For comparison purposes, the voltage sensitivity is expressed here in $\Omega / \mathrm{T} / \mathrm{m}$ after dividing it by the excitation current amplitude. These voltage sensitivity values correspond to those of Fig. 7 for $I_{a c}=1 \mathrm{~mA}_{\text {rms }}$ simply expressed in $\Omega / \mathrm{T} / \mathrm{m}$ instead of $\mathrm{V} / \mathrm{T} / \mathrm{cm})$. The agreement between the two measurements indicates that all samples are still operating in their linear ranges for excitation current amplitudes below $1 \mathrm{~mA}_{\mathrm{rms}}$. Then, the maximum voltage sensitivity, $S_{\mathrm{V} / \mathrm{T}_{\text {max }}}$, at $f=3 \mathrm{MHz}$, as given in Table 3 , is also presented as curve (c). Clearly, the order of increasing sensitivity would be different if the criterion were $S_{\mathrm{V} / \mathrm{T}_{\max }}$ rather than $S_{\Omega / \mathrm{T}_{\max }}$. Note that the difference in (c) is smaller between samples than in (a) and (b). As discussed in the Introduction, the intrinsic impedance sensitivity measured in the linear regime is not necessarily a good metric for the maximum voltage sensitivity, which is the relevant parameter for highly sensitive magnetometry. This is illustrated in Figs. 7 and 8 . The intrinsic impedance depends, at least part upon the anisotropy, which is affected by residual strains. The maximum voltage sensitivity is critically affected by the maximum driving current, beyond which sensitivity decreases. The behavior of these quantities in non-ideal wires is not entirely clear.

We now compare, in Fig. 9, the measured optimal voltage sensitivity and that anticipated from Eq. (3a), for a frequency of $10 \mathrm{MHz}$. A stiffness constant $A=10^{-11} \mathrm{~J} / \mathrm{m}$ was assumed. We have previously shown that wires similar to those of series "a" exhibit GMI peaks considerably smaller than predicted by the linear model for ideal cylinders, both in the linear [20, 21] and non-linear [18] regimes. It is therefore not surprising that the line deduced from Eq. (3a) overestimates the experimental values for the optimum sensitivity, as well.

While Eq. (3a) (as well as Eq. (3b)) systematically overestimates the experimental values, as indicated by the red line in Fig. 9, it provides frame-

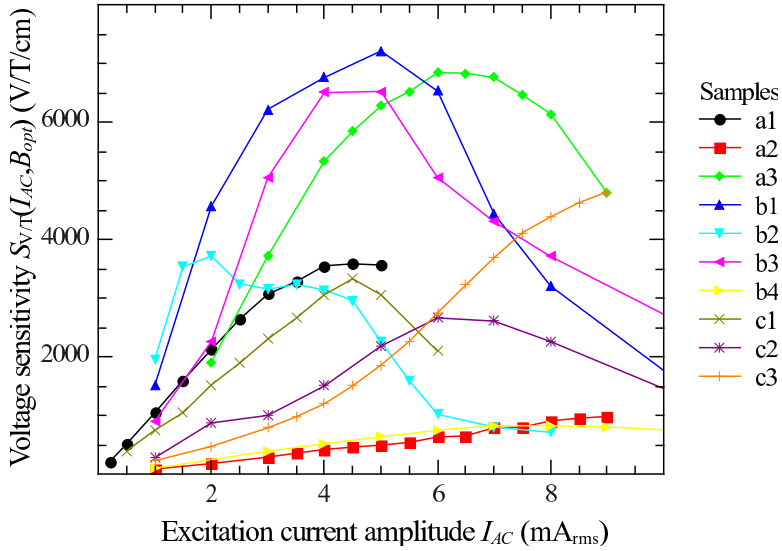

Figure 7: Highest voltage sensitivity of each sample as a function of excitation current amplitude, at an operating frequency of $3 \mathrm{MHz}$ and sample length of $1.5 \mathrm{~cm}$. All samples were DC biased by their respective optimal DC bias currents, evaluated from impedance measurement in the linear range. Optimal excitation current is never reached for samples a2 and $\mathrm{c} 3$.
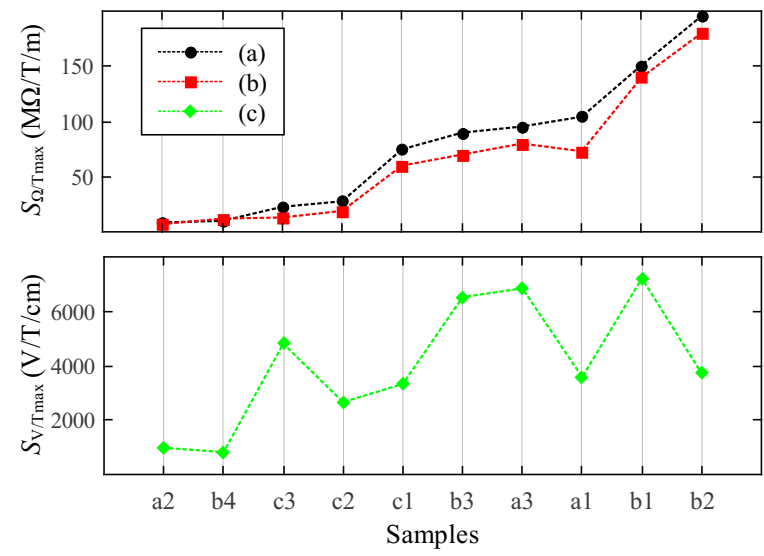

Figure 8: Comparison of intrinsic and voltage sensitivities of each sample measured at an excitation frequency of $3 \mathrm{MHz}$. Connecting lines are only used as a guide for the eye. Curve (a): the maximum intrinsic impedance sensitivity, $S_{\Omega / \mathrm{T}_{\max }}$, obtained from impedance measurement in the linear range. Curve (b): voltage sensitivity, $S_{\mathrm{V} / \mathrm{T}}\left(I_{A C}, B_{o p t}\right)$, for an excitation current $I_{A C}$ of $1 \mathrm{~mA}_{\mathrm{rms}}$ divided by the amplitude of this current. Both (a) and (b) curves are expressed in $\Omega / \mathrm{T} / \mathrm{m}$. The maximum voltage sensitivity, $S_{\mathrm{V} / \mathrm{T}_{\max }}$, obtained for the optimal excitation current amplitude, $I_{A C-o p t}$, is given by curve (c), expressed in $\mathrm{V} / \mathrm{T} / \mathrm{cm}$. Sample list of the abscissa axis is ordered by increasing intrinsic impedance sensitivity, curves (a), which highlights the importance of the permissible optimal excitation current amplitude on voltage sensitivity performance, curve (c). 
Table 3: Maximum voltage sensitivity obtained for each $1.5 \mathrm{~cm}$ long sample, with the corresponding optimal operating conditions. Values in italics correspond to those for which the optimal driving current amplitude is not reached (greater than $10 \mathrm{~mA})$.

\begin{tabular}{|c|c|c|c|c|c|c|c|c|c|}
\hline \multicolumn{2}{|c|}{ Operating frequency } & \multicolumn{2}{|c|}{$300 \mathrm{kHz}$} & \multicolumn{2}{|c|}{$1 \mathrm{MHz}$} & \multicolumn{2}{|c|}{$3 \mathrm{MHz}$} & \multicolumn{2}{|c|}{$10 \mathrm{MHz}$} \\
\hline Sample & $\begin{array}{l}I_{D C^{\mathrm{i}}} \\
\mathrm{mA}_{\mathrm{dc}}\end{array}$ & $\begin{array}{c}S_{\mathrm{V} / \mathrm{T}_{\max }} \\
\mathrm{kV} / \mathrm{T}\end{array}$ & $\begin{array}{c}I_{A C-o p t} \\
\mathrm{~mA}_{\mathrm{rms}}\end{array}$ & $\begin{array}{c}S_{\mathrm{V} / \mathrm{T}_{\max }} \\
\mathrm{kV} / \mathrm{T}\end{array}$ & $\begin{array}{c}I_{A C-o p t} \\
\mathrm{~mA}_{\mathrm{rms}}\end{array}$ & $\begin{array}{c}S_{\mathrm{V} / \mathrm{T}_{\max }} \\
\mathrm{kV} / \mathrm{T}\end{array}$ & $\begin{array}{c}I_{A C-o p t} \\
\mathrm{~mA}_{\mathrm{rms}}\end{array}$ & $\begin{array}{c}S_{\mathrm{V} / \mathrm{T}_{\max }} \\
\mathrm{kV} / \mathrm{T}\end{array}$ & $\begin{array}{c}I_{A C-o p t} \\
\mathrm{~mA}_{\mathrm{rms}}\end{array}$ \\
\hline$\overline{\mathrm{a} 1}$ & 0.5 & 1.03 & 2 & 2.76 & 3 & 5.37 & 4.5 & 6.93 & $>10$ \\
\hline $\mathrm{a} 2$ & 6 & 0.49 & $>10$ & 0.78 & $>10$ & $1.4^{7}$ & $>10$ & 1.50 & $>10$ \\
\hline $\mathrm{a} 3$ & 0.5 & 3.02 & 3.5 & 6.84 & 5 & 10.26 & 6 & 15.2 & $>10$ \\
\hline b1 & 2 & 1.58 & 3 & 5.40 & 4 & 10.81 & 5 & 16.27 & 7 \\
\hline $\mathrm{b} 2$ & 0 & 1.39 & 1 & 3.77 & 1.5 & 5.57 & 2 & 8.91 & 6 \\
\hline b3 & 2 & 1.42 & 3 & 4.65 & 4 & 9.78 & 5 & 14.58 & 6 \\
\hline $\mathrm{b} 4$ & 2 & 0.16 & 5 & 0.47 & 6 & 1.23 & 8 & 4.19 & 9 \\
\hline $\mathrm{c} 1$ & 0 & 0.80 & 1.5 & 1.86 & 3.5 & 4.99 & 4.5 & 9.67 & 9 \\
\hline $\mathrm{c} 2$ & 0.5 & 0.47 & 3 & 1.35 & 5 & 3.99 & 6 & 9.29 & $>10$ \\
\hline c3 & 6 & 1.27 & $>10$ & 3.47 & $>10$ & 7.21 & $>10$ & 7.99 & $>10$ \\
\hline
\end{tabular}

${ }^{\mathrm{i}}$ The optimal DC bias current is deduced from intrinsic impedance sensitivity characterization as discussed in Sec. 2.4 .

work for interpreting the data. Based on Eq. (3a), which applies to ideal samples, all CoFe-rich samples (all except a2) have a predicted sensitivity around 10 to $15 \mathrm{kV} / \mathrm{T} / \mathrm{cm}$, with a corresponding large spread of measured values. Considering that the error bars in the prediction are large, primarily due to the uncertainty in the determination of the resistivity, and that several samples were not operated under optimal conditions, it is rather difficult to establish a clear trend from this set of data. Using Eq. (3b), which could in principle apply to non-ideal wires, does not improve the correlation. Indeed, using Gilbert damping parameters determined at $38 \mathrm{GHz}$ to predict the response at $10 \mathrm{MHz}$ or below is somewhat questionable, as discussed below. Finally, both Eqs. (3a) and (3b) predict that the sensitivity would be higher in materials with higher magnetization and resistivity. While this may partly explain the poor sensitivity of the $\mathrm{NiCo}-$ based sample, which has the highest magnetic susceptibility of them all but the lowest $M_{s} \rho$ product, this general trend is probably hidden by the effect of inhomogeneity in the samples.

In Fig. 10, we compare the frequency dependence observed for $S_{\mathrm{V} / \mathrm{T}_{\max }}$ for the three best samples, representative of each fabrication process (a3, b1 and $\mathrm{c} 1$ ). The observed sensitivity increases with frequency, but is always lower, than the $\omega^{1 / 4}$ dependence predicted by (3a) for an ideal wire. We suggest that the deviations from ideal wire behavior, shown in Figs. 9 and 10, are related to wire de- fects and imperfections as discussed in Sec. 2.2, in relation to the approach to saturation. As discussed previously [2], the GMI of non-ideal samples can often be modeled using $\alpha$ as an effective frequency dependent phenomenological damping parameter including both intrinsic and extrinsic damping mechanisms. Such damping is expected to be higher at low frequency (and therefore low applied field) where the effect of inhomogeneities is the most significant. The $\alpha$-values reported in Table 2 were measured at $38 \mathrm{GHz}$ under applied magnetic fields which are three orders of magnitude higher than those used at the operating GMI points. As such, they are not necessarily representative of the effective values of $\alpha$ at low field, but rather indicative of the potential sensitivity of ideal wires.

\section{Discussion and conclusion}

The GMI sensitivity of several rapidly solidified soft microwires has been studied and analyzed using a simple phenomenological model proposed in Ref. [2]. An important objective of our study was to find a way of meaningfully comparing wires with different radius and exhibiting different physical properties. We propose that voltage sensitivity per unit length of wire, measured under optimal driving current, which is directly related to magnetic sensor performance, is a critical metric to assess the wire potential for sensitive magnetometry. In this respect, we found no evidence permitting us to iden- 


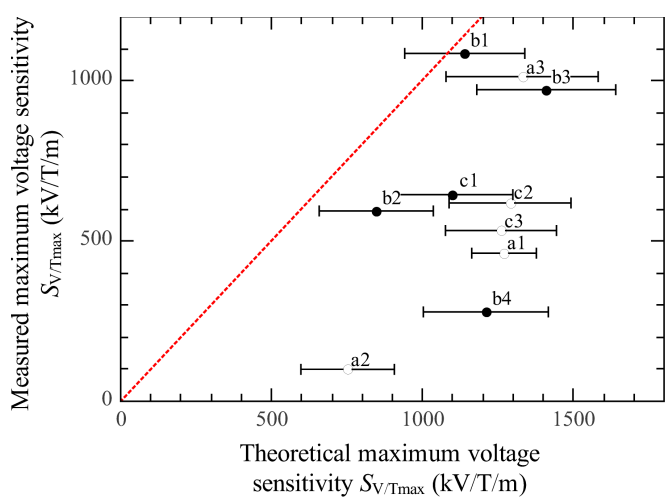

Figure 9: Comparison of measured and theoretical maximum voltage sensitivity, $S_{\mathrm{V} / \mathrm{T}_{\text {max }}}$, expressed in $\mathrm{kV} / \mathrm{T} / \mathrm{m}$, for an excitation frequency of $10 \mathrm{MHz}$. The red line illustrates a match between measurement and theory (i.e. measured values are equal to theoretical ones). Sample references are shown for each point and empty symbols are used when the optimal excitation amplitude is not reached since it is higher than $10 \mathrm{~mA}_{\text {rms }}$.

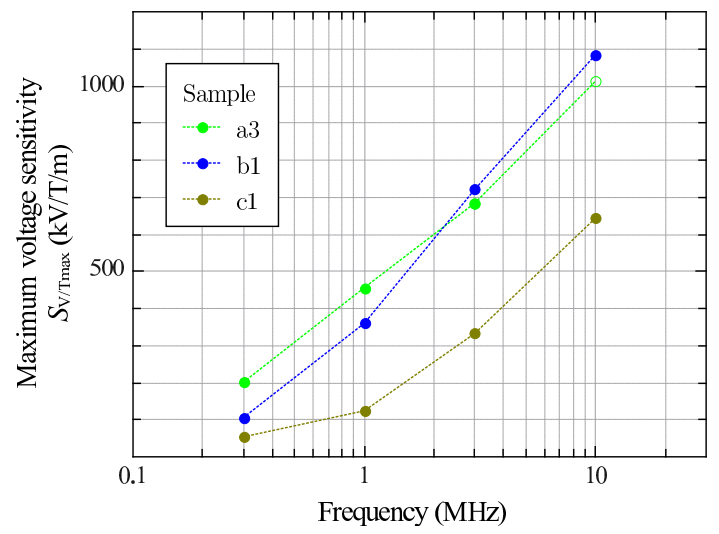

Figure 10: Measured maximum voltage sensitivity, expressed in $\mathrm{kV} / \mathrm{T} / \mathrm{m}$, for samples $\mathrm{a} 3, \mathrm{~b} 1$ and $\mathrm{c} 1$, as a function of the excitation frequency, $f$. Empty symbols are used when the optimal excitation current amplitude is not reached since it is higher than $10 \mathrm{~mA}_{\mathrm{rms}}$. tify a sample which greatly surpasses all the others. Further, the variability of samples from a given source appears to be comparable with the variability between sources. In any case, the best samples still exhibit sensitivities significantly below the prediction for perfectly cylindrical material with free surface spins and without defects or nonuniformities. This suggests that no microwires currently fabricated approach ideal properties.

A methodology based on VSM magnetometry, ferromagnetic resonance spectroscopy and fourpoint resistance measurements, has been employed to determine various physical properties entering into the model. As highlighted, measurement of magnetic moment divided by the sample volume was found to be unreliable to determine the saturation magnetization. Therefore, an alternative approach has been proposed. Overall, the physical properties of different samples (in some cases, nominally identical), exhibit significant fluctuations. Nonetheless, the dispersion of these physical properties between samples with similar compositions appears to be real, thus opening the question of the physical uniformity of rapidly solidified soft amorphous wires. Despite considerable effort to control these, measurements of intrinsic magnetoimpedance sensitivity sometimes suffer from problems of reproducibility of electrical contacts and of mechanical stresses introduced during the contact procedure. According to the model presented here, change of sensitivity due to unintentional stress (induced magnetoelastic anisotropy) can be somewhat compensated by adjusting the optimal driving current. The reproducibility of electrical contacts can also be significantly improved by electroplating $\mathrm{Cu}$ on the contact zone.

As expected from earlier experiments and from nonlinear modeling [18], there exists an optimum voltage sensitivity (at a given frequency), at a current slightly above that at which nonlinear behavior is first observed. The optimal operating conditions are specific to each sample (and sensitive to the contact and stress conditions). When they were operated under these conditions, the sensitivity of the microwires approached the predictions of the model more closely and the variability from sample to sample was considerably reduced. While the frequency dependence of optimal sensitivity (Fig. 10) is similar for all samples, the differences are sufficient to modify the order of sensitivities. In different sets of operating conditions (driving current amplitude and frequency) different wires exhibited 
the best performances.

It is important to realize that high impedance sensitivity in the linear regime is not a guarantee of maximum voltage sensitivity in a given set of conditions. While low anisotropy and low damping lead to high intrinsic sensitivity materials, they are likely to adversely affect the optimum voltage sensitivity by reducing the critical driving current above which the sensitivity starts to decrease. Samples with higher anisotropy may be able to reach the same sensitivity because of their higher values of $H_{\text {crit }}$. This is offset, of course, by the practical limits to the currents to be employed, as discussed above.

While the model presented here provides a useful framework to analyze the GMI data, several important details are still hidden in some parameters, such as $H_{\text {crit }}$, which relates to the optimal driving current, and $H_{p k}$. An important factor to consider in detail is the frequency dependence of the $H_{c r i t} / H_{p k}$ term of Eqs. (3a) and (3b). At relatively low frequencies, $H_{p k}$ is approximately constant, while $H_{c r i t}$ increases significantly. Thus, optimal sensitivity increases with frequency up to a maximum value, after which it decreases, due to the increase in $H_{p k}$. This has two important consequences. First, the optimal driving current, $H_{c r i t}$, eventually exceeds practical values to be used in magnetometer circuits. Here, we have been limited to $\mathrm{AC}$ currents of $10 \mathrm{~mA}$ by the electronics, so that half of the samples had not reached their optimal sensitivity at $10 \mathrm{MHz}$. Second, the variation of $H_{c r i t} / H_{p k}$ is surely affected by material parameters and various kinds of defects. The nature of this dependence is not yet fully understood, but it is currently under study. From nonlinear modeling [18], it is expected that the onset of non-linear behavior, which ultimately limits the optimal drive current, will occur at lower currents for material with lower damping and lower anisotropy.

The fact that the predicted correlation of the sensitivity to magnetization and resistivity is far from obvious in the experimental data is not entirely surprising. Apart from the uncertainties in the determination of the physical parameters, none of the samples are ideal wires. Thus, differences between theoretical and experimental values also arise from deviations from ideality. Stress distribution and defects, associated with the non-ideal approach to saturation of all the samples studied, are most likely to affect $H_{c r i t}$ and the effective damping, expressed here as an effective Gilbert coefficient $\alpha$. In such circumstances, Eq. (3b) might be more appropriate than Eq. (3a), even at low frequency, provided one uses a field and frequency dependent damping parameter, which is unknown a priori.

\section{Acknowledgments}

The authors are indebted to Horia Chiriac and Arkady Zhukov who kindly provided samples for this study. Special thanks are due to LouisPhilippe Carignan, Christian Lacroix, and Vincent Boucher for valuable discussion and help during physical properties characterization and to Dr. Djamel Seddaoui for helpful suggestions on the manuscript. This work was supported in part by a mobility award granted by Frontenac program for France-Quebec exchange and by grants from CRSNG (Canada).

[1] M. Knobel, M. Vázquez, L. Kraus, Giant magnetoimpedance, Handbook of magnetic materials 15 (2003) 497-563.

[2] D. Ménard, D. Seddaoui, L. G. C. Melo, A. Yelon, B. Dufay, S. Saez, C. Dolabdjian, Perspectives in Giant Magnetoimpedance Magnetometry, Sensor Letters 7 (3) (2009) 339-342.

[3] L. Ding, S. Saez, C. Dolabdjian, L. G. C. Melo, A. Yelon, D. Ménard, Equivalent Magnetic Noise Limit of Low-Cost GMI Magnetometer, IEEE Sensors Journal 9 (2) (2009) 159-168.

[4] L. G. C. Melo, D. Ménard, A. Yelon, L. Ding, S. Saez, C. Dolabdjian, Optimization of the magnetic noise and sensitivity of giant magnetoimpedance sensors, Journal of Applied Physics 103 (3) (2008) 033903-033903.

[5] J. O. Strom-Olsen, P. Z. Rudkowski, Ferromagnetic fibers having use in electronic article surveillance and method of making same, US Patent 5003291 (1991)

[6] H. Chiriac, T. A. Óvári, Amorphous glass-covered magnetic wires: preparation, properties, applications, Progress in materials Science 40 (5) (1996) 333-407.

[7] I. Ogasawara, S. Ueno, Preparation and properties of amorphous wires, IEEE Transactions on Magnetics 31 (2) (1995) 1219-1223.

[8] L.-P. Carignan, C. Lacroix, A. Ouimet, M. Ciureanu, A. Yelon, D. Menard, Magnetic anisotropy in arrays of $\mathrm{Ni}, \mathrm{CoFeB}$, and $\mathrm{Ni} / \mathrm{Cu}$ nanowires, Journal of Applied Physics 102 (2) (2007) 023905.

[9] J. M. D. Coey, Magnetism and magnetic materials, Cambridge University Press, 2010.

[10] L. Néel, La loi d'approche en a: H et une nouvelle théorie de la dureté magnétique, Journal de Physique et le Radium 9 (5) (1948) 184-192.

[11] W. Brown, The Effect of Dislocations on Magnetization Near Saturation, Physical Review 60 (2) (1941) 139147.

[12] H. Kronmüller, Magnetization processes and the microstructure in amorphous metals, Le Journal de Physique Colloques 41 (C8) (1980) 618-625.

[13] L. Kraus, Theory of ferromagnetic resonances in thin wires, Czechoslovak Journal of Physics 32 (11) (1982) $1264-1282$. 
[14] C. Kittel, On the theory of ferromagnetic resonance absorption, Phys. Rev. 73 (2) (1948) 155-161.

[15] J. H. Van Vleck, Ferromagnetic resonance, Physica 17 (3-4) (1951) 234-252.

[16] V. Boucher, D. Ménard, Effective magnetic properties of arrays of interacting ferromagnetic wires exhibiting gyromagnetic anisotropy and retardation effects, Physical Review B 81 (17) (2010) 1-21.

[17] B. Dufay, S. Saez, C. Dolabdjian, D. Seddaoui, A. Yelon, D. Ménard, Improved GMI Sensors Using Strongly-Coupled Thin Pick-Up Coils, Sensor Letters 7 (3) (2009) 334-338.

[18] D. Seddaoui, D. Ménard, B. Movaghar, A. Yelon, Nonlinear electromagnetic response of ferromagnetic metals: Magnetoimpedance in microwires, Journal of Applied Physics 105 (8) (2009) 083916.

[19] P. Garoche, A. P. Malozemoff, Approach to magnetic saturation in sputtered amorphous films: Effects of structural defects, microscopic anisotropy, and surface roughness, Physical Review B 29 (1) (1984) 226-231.

[20] D. Ménard, A. Yelon, Theory of longitudinal magnetoimpedance in wires, Journal of Applied Physics 88 (2000) 379

[21] L. G. C. Melo, D. Ménard, P. Ciureanu, A. Yelon, Influence of surface anisotropy on magnetoimpedance in wires, Journal of applied physics 92 (12) (2002) 72727280 . 